\title{
Quantum work and information geometry of a quantum Myers-Perry black hole
}

\author{
Behnam Pourhassan, ${ }^{a, b}$ Salman Sajad Wani, ${ }^{b}$ Saheb Soroushfar ${ }^{c}$ and Mir Faizal ${ }^{d, e, b}$ \\ ${ }^{a}$ School of Physics, Damghan University, \\ Damghan, 3671641167, Iran \\ ${ }^{b}$ Canadian Quantum Research Center, \\ 204-3002 32 Ave Vernon, BC V1T 2L7 Canada \\ ${ }^{c}$ Faculty of Technology and Mining, Yasouj University, \\ Choram 75761-59836, Iran \\ ${ }^{d}$ Department of Physics and Astronomy, University of Lethbridge, \\ Lethbridge, Alberta, T1K 3M4, Canada \\ ${ }^{e}$ Irving K. Barber School of Arts and Sciences, University of British Columbia, \\ Kelowna, British Columbia, V1V 1 V7, Canada \\ E-mail: b.pourhassan@du.ac.ir, salmansajadwani@gmail.com, \\ soroush@yu.ac.ir, mirfaizalmir@googlemail.com
}

ABSTRACT: In this paper, we will obtain quantum work for a quantum scale five dimensional Myers-Perry black hole. Unlike heat represented by Hawking radiation, the quantum work is represented by a unitary information preserving process, and becomes important for black holes only at small quantum scales. It will be observed that at such short distances, the quantum work will be corrected by non-perturbative quantum gravitational corrections. We will use the Jarzynski equality to obtain this quantum work modified by non-perturbative quantum gravitational corrections. These non-perturbative corrections will also modify the stability of a quantum Myers-Perry black hole. We will define a quantum corrected information geometry by incorporating the non-perturbative quantum corrections in the information geometry of a Myers-Perry black hole. We will use several different quantum corrected effective information metrics to analyze the stability of a quantum Myers-Perry black hole.

KeYwords: Black Holes, Models of Quantum Gravity

ARXiv EPRINT: 2102.03296 


\section{Contents}

1 Introduction 1

2 Non-perturbative corrections 3

3 Quantum work 5

4 Information geometry 11

$\begin{array}{llr}5 & \text { Conclusion } & 18\end{array}$

\section{Introduction}

It is known that black holes are maximum entropy objects, and this maximum entropy of black holes scales with the area of their horizon $[1,2]$. So, the maximum entropy of a region scales with its area rather than its volume. This scaling of maximum entropy with the area of a region has motivated the development of the holographic principle [3, 4]. It has been suggested that the relation between area and entropy can be modified due to quantum gravitational corrections $[5,6,8]$. However, as the corrected entropy is still a function of the area of the horizon, these corrections can also be investigated using the holographic principle $[9,10]$. In fact, as AdS/CFT correspondence is a concrete realization of the holographic principle, it has been used to obtain such quantum corrections to the entropy of black holes [11-15]. Such quantum correction have also been obtained using extremal limit of black holes $[16,17]$. The quantum corrections to the entropy of a black hole can be obtained from a conformal field theory, using the density of microstates associated with conformal blocks [18]. As the Cardy formula can be used to obtain the entropy of a conformal field theory, it has been used to calculate the quantum corrections to the black hole entropy [19]. The quantum corrected entropy of a black hole has also been obtained using the Rademacher expansion [20]. Thus, various different approaches have been used to obtain quantum corrected entropy of black holes.

The geometry of space-time emerges from thermodynamics in the Jacobson formalism [21]. So, it is expected that quantum fluctuation of space-time can be obtained from the thermal fluctuations in the Jacobson formalism [22]. As it is possible to obtain the perturbative thermal fluctuations to the thermodynamics of various black holes [23-27], it has been argued that such perturbative thermal fluctuations can be used to obtain perturbative quantum fluctuations [21, 22]. In fact, perturbative quantum corrections to various black holes have been constructed using the perturbative thermal fluctuations [28-31]. As the temperature of black holes scales inversely with its size, the thermal fluctuations can be neglected for large black holes. Since the quantum fluctuations can be obtained using thermal fluctuations [21, 22], we can also neglect the quantum fluctuations of large black holes. However, these quantum corrections become important for smaller black holes, and 
they cannot be neglected after a certain scale. The small perturbative quantum corrections to various black holes can be obtained using thermal perturbations near the equilibrium [28-31]. These perturbative corrections are valid for black holes, where the thermal fluctuations are small enough for them to be expressed as small perturbations near the equilibrium. Thus, they are expressed in terms of the original equilibrium entropy and temperature [23-27]. However, for black holes whose size is comparable to Planck scale, the corrections to the entropy cannot be described as perturbative corrections near the equilibrium. At such a short distance, we need to consider the full non-perturbative quantum gravitational corrections to the thermodynamics of black holes. It is expected that such non-perturbative quantum gravitational corrections produce non-trivial modifications to the entropy of black holes. In fact, it has been demonstrated that the corrected entropy of such quantum black holes can be expressed as an exponential function of the original entropy [32]. Such non-perturbative corrections to the entropy of a black hole have also been obtained from the Kloosterman sums [33]. To obtain such non-perturbative corrections, the near horizon behavior of mass-less supergravity fields was investigated using the AdS/CFT correspondence [34, 35]. Now, as these non-perturbative corrections [32, 33] have been analyzed using string theoretical effects $[34,35]$, it is important to investigate such nonperturbative corrections to the extra dimensional geometries motivated by string theory.

As string theory is only consistent in extra dimensions, it is possible to motivate the study of extra dimensional black holes from it. So, using such string theoretical motivation, it is possible to study a five dimensional Myers-Perry black hole [36]. The Myers-Perry black hole rotates in two independent planes, and can be considered as a generalization of the Kerr black hole to extra dimensions. The thermodynamics of such a five dimensional Myers-Perry black hole has been investigated [37, 38]. The effect of perturbative quantum corrections to the thermodynamics of the Myers-Perry black hole has also been studied [40]. So, we will analyze the effects of non-perturbative corrections to a five dimensional quantum Myers-Perry black hole. As these non-perturbative corrections cannot be analyzed as small fluctuations near the equilibrium, it is important to use non-equilibrium quantum thermodynamics to analyze them [60-63]. It is possible to define a quantum analog of classical work in quantum thermodynamics. It may be noted that as quantum work is associated with a quantum process, it cannot be obtained using a single measurement of a quantum observable. However, it possible to obtain quantum work using the quantum Crooks fluctuation theorem, by making two measurements [64, 65]. As it is possible to obtain Jarzynski equality from quantum Crooks fluctuation theorem, the Jarzynski equality has been used to relates quantum work with the difference of equilibrium free energies [66, 67]. Now as we can obtain the equilibrium free energies for a black hole, it can be used to obtain information about the quantum work done during its evaporation. Thus, the Jarzynski equality has been used to obtain quantum work for black holes [68, 69]. It may be noted that here quantum work is obtained from the original Bekenstein-Hawking entropy of the black holes. This original Bekenstein-Hawking entropy is obtained from semi-classical approximations by using quantum field theory in curved space-time $[1,2]$. Hence, for the original Bekenstein-Hawking entropy quantum gravitational effects are neglected. However, at such scales, quantum gravitational corrections cannot be neglected, and we need to 
consider the non-perturbative quantum gravitational corrections $[32,33]$ to quantum work. So, in this paper, we will analyze the non-perturbative quantum gravitational corrections to the quantum work of a Myers-Perry black hole during its evaporation. It may be noted that the information geometry of the Myers-Perry black hole has also been studied using different information metrics [41]. We will analyze the non-perturbative quantum gravitational corrections to the information geometry of the Myers-Perry black hole, by defining quantum corrected effective informational metrics.

\section{Non-perturbative corrections}

In this section, we will analyze the non-perturbative corrections to a Myers-Perry black hole. The Myers-Perry black hole is a five dimensional black hole, which rotates in two independent planes. The metric for such a five dimensional Myers-Perry black hole can be expressed as (in Planck units) [36, 37],

$$
\begin{aligned}
d s^{2}= & -d t^{2}+\frac{\Sigma r^{2}}{\Delta} d r^{2}+\Sigma d \theta^{2}+\left(r^{2}+a^{2}\right) \sin ^{2} \theta d \varphi^{2} \\
& +r^{2} \cos ^{2} \theta d \zeta^{2}+\frac{\mu}{\Sigma}\left(d t-a \sin ^{2} \theta d \varphi\right)^{2}
\end{aligned}
$$

where $a$ is angular momentum and $\mu$ is mass parameter. It may be noted that usually the general form of Myers-Perry black hole solution is parameterized by $m, a_{1}, a_{2}$, and here we have taken $m=\mu, a_{1}=a, a_{2}=0$, for simplification. Here $\Sigma=r^{2}+a^{2} \cos ^{2} \theta$, and $\Delta=r^{2}\left(r^{2}+a^{2}-\mu\right)$, are suitable functions in the metric [37]. It is possible to express the radius of the horizon in terms of $\mu$ which is a parameter related to mass, and $a$ which is a parameter related to the angular momentum, as $r_{h}=\sqrt{\mu-a^{2}}$. The explicit mass and angular momentum of the Myers-Perry black hole can expressed in terms of $\mu$ and $a$ as $M=3 \mu / 8$ and $J=2 a M / 3$. We have expressed the Myers-Perry black hole using $(M, J)$ as the non-perturbative corrections to the information geometry can be directly obtained using $(M, J)$.

The surface gravity of these solutions given by [42],

$$
\kappa=\frac{r_{h}}{r_{h}^{2}+a^{2}} .
$$

The temperature of the Myers-Perry black hole can now be written as $T=\kappa / 2 \pi[42]$. By using the horizon radius, the Myers-Perry black hole temperature can obtain in terms of mass and angular momentum as,

$$
T=\frac{\sqrt{96 M^{3}-81 J^{2}}}{32 \pi^{2} M^{2}} .
$$

In figure 1 we can see behavior of temperature in terms of $M$ by variation of the angular momentum. We can see that there is a lower limit for $M$ in presence of $J$.

The rotation parameter has an upper bound, which is given by $a<\sqrt{8 M / 3}$. Now for quantum sized black holes, we can write $M<1$. So, using $J^{2}<\frac{96}{81} M^{3}$, for quantum sized black hole (with $M<1$ ), we observe $J \ll 1$. It may be noted that the entropy of a back 


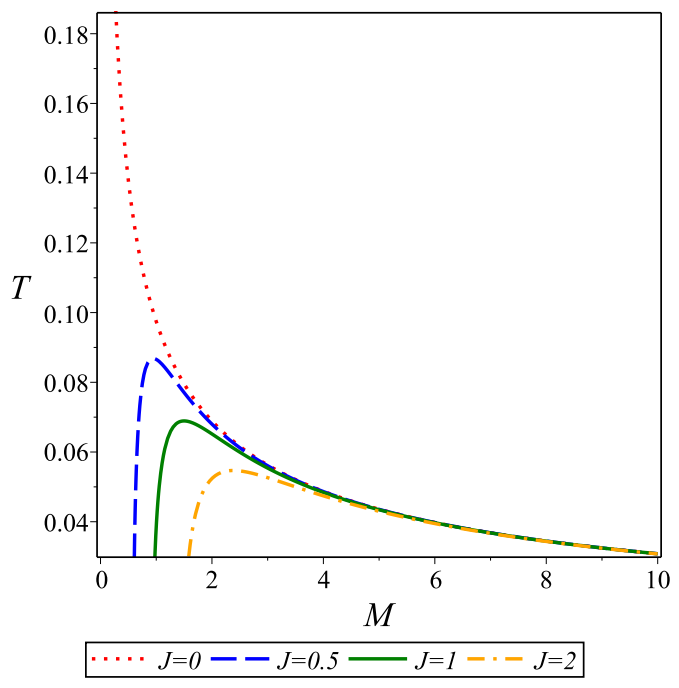

Figure 1. Temperature of the Myers-Perry black hole in terms of mass.

hole can be obtained from microstates of a conformal field theory [52-55]. The microstates of black holes with angular momentum have been studied, and it has been observed that they can be described as a function of its mass and angular momentum $\Omega(M, J)$ [56-59]. So, we can write the entropy of a Myers-Perry black hole in terms of its microstates as

$$
S_{0}=\ln \Omega(M, J)=\frac{2 \pi^{2} \sqrt{96 M^{3}-81 J^{2}}}{9} .
$$

This original entropy of the Myers-Perry black hole will get modified by quantum gravitational corrections at short distances. This is because the relation between entropy and area of the horizon of any black hole (including a Myers-Perry black hole) is expected to be modified due to the quantum gravitational corrections at short distances. It may be noted that in the Jacobson formalism, geometry of space-time emerges from thermodynamics [21], and so these quantum corrections can be obtained from the thermal fluctuations [22]. In fact, perturbative thermal fluctuations have been used to obtain perturbative quantum corrections to the geometry of black holes [28-31]. Such corrections are universal, and can be obtained from microstates of a conformal field theory [52-55]. It has been demonstrated that perturbative correction to the entropy of a black hole can be obtained from modular invariance. of the partition functions for a conformal field theory [52-55], and scales as $[23-27]$

$$
S_{\text {per }}=f(\Omega) \sim \ln \left(S_{0}\right)+\ldots,
$$

where $S_{0}$ is the original equilibrium entropy of the black hole. These corrections to the original equilibrium entropy have been obtained by analyzing small fluctuations around the equilibrium. This is done by first noting that from modular invariance of the partition function for the conformal field theory [52-55], we can write $S(\beta)=a \beta^{n}+b \beta^{m}$, with the constant $a, b, n, m,>0$. Now as $S(\beta)$ has an extremum $\beta_{0}=(n b / m a)^{1 / m+n}=T^{-1}$, these perturbations can be expressed in terms of the original equilibrium entropy $S_{0}=S\left(\beta_{0}\right)$ 
(with the original equilibrium temperature $T=1 / \beta_{0}$ ) [23-27]. These perturbative corrections become important when the black hole is small, and can be neglected when it is very large. However, as the black hole reduces further, this perturbative description does not hold, and we need to consider non-equilibrium corrections to the thermodynamics of black holes. These non-equilibrium corrections will correspond to non-perturbative quantum corrections to the geometry of black holes. Such non-perturbative corrections would modify the original entropy of a black hole. In fact, it has been argued that the non-perturbative corrections to the black hole would scale as [32-35]

$$
S_{m}=\frac{1}{\Omega} \sim e^{-S_{0}} .
$$

So, we will analyze the effects of such non-perturbative corrections [32-35] on a MyersPerry black hole. We can express the corrected entropy of a Myers-Perry black hole as $S=S_{0}+\eta e^{-S_{0}}$, by introducing a parameter $\eta$. This parameter controls the strength of the non-perturbative corrections, and has been motivated from the use of such a control parameter in perturbative corrections [23-27]. The value of $\eta$ is fixed in such a way that for a large black hole, these corrections are negligible, and they only change the behavior of thermodynamics for small quantum scale Myers-Perry black holes. So, we can explicitly write the non-perturbatively corrected entropy for such a small quantum scale Myers-Perry black holes as

$$
S=\frac{2 \pi^{2} \sqrt{96 M^{3}-81 J^{2}}}{9}+\eta \exp \left(-\frac{2 \pi^{2} \sqrt{96 M^{3}-81 J^{2}}}{9}\right) .
$$

This exponential non-perturbative quantum correction to the original entropy of a MyersPerry black hole is neglected at large distances. It has been argued that perturbative correction to the entropy can produce interesting modifications for the thermodynamic behavior of the system [23-27]. Thus, we expect that the non-perturbative correction [3235 ] would also produce non-trivial modifications to the quantum thermodynamics of this system. So, we will be using the non-equilibrium quantum thermodynamics [60-63] to analyze such a system, as at such small quantum scales, with non-perturbative quantum corrections, non-equilibrium description has to be used to study a quantum Myers-Perry black hole.

\section{Quantum work}

In previous section, we have analyzed the quantum gravitational corrections to a quantum sized Myers-Perry black hole. We also observed that at such a scale, we cannot express the system as a equilibrium system, and we need to use non-equilibrium quantum thermodynamics to investigate it. It is known that the quantum work is one of the most important quantities in quantum thermodynamics [60-63]. The quantum work can be obtained from the quantum Crooks fluctuation theorem using Jarzynski equality [64, 65]. The Jarzynski equality relates the non-equilibrium measurements of the work done on a system to difference between equilibrium free energies. As it is possible to calculate the free energies between two thermodynamical states of a black hole, the quantum work done during the 
evaporation of a black holes has also been obtained using the Jarzynski equality [68, 69]. Even though the non-equilibrium thermodynamics of black holes has been previously studied $[68,69]$, the quantum gravitational corrections [32, 33] to the non-equilibrium thermodynamics were neglected in those investigations. However, at such small scales, at which the system cannot be described as an equilibrium system, we cannot neglect quantum gravitational corrections to it $[32,33]$. Hence, we investigate the effect of such quantum gravitational corrections $[32,33]$ on quantum work done during the evaporation of the Myers-Perry black hole.

As the Myers-Perry black hole evaporates, the number of its microstates changes. So, a Myers-Perry black hole with initial microstates $\Omega\left(M_{2}, J_{2}\right)$ can evaporate to a MyersPerry black hole with microstates $\Omega\left(M_{1}, J_{1}\right)$. This change is produced due to Hawking radiation, whose temperature scales with surface gravity as $T=\kappa / 2 \pi$ [42]. Now when the black hole is large, its temperature is small, and the rate of change of microstates is much smaller than the original microstates. However, as the black hole becomes small, its temperature increases, and the rate of change of mircostates also increases. Furthermore, as the microstates are proportional to the entropy, the total number of microstates also decreases as the black hole becomes small. Thus, at a sufficient small size, we cannot neglect the effect of the rate of change of microstates, and at this stage, we cannot consider the system in equilibrium. It may be noted that for Myers-Perry black hole, large is defined in a thermodynamic sense, as the microstates are functions of both mass and angular momentum, and their combined effect defines the size of thermodynamic system. So, for small quantum sized black holes, we have to explicitly analyze the effects of such changes between two thermodynamic states, and use non-equilibrium quantum thermodynamics to analyze it $[68,69]$.

As the microstates are related to the entropy, at quantum scales, we need to investigate the change in the entropy of the Myers-Perry black hole due to a change in its microstates. Now at small scales, we have to also consider the effects of non-perturbative quantum gravitational corrections to the entropy of such a Myers-Perry black hole [32-35]. So, we can express the change in the quantum gravitationally corrected entropy of a Myers-Perry black hole as $\Delta S=S\left(M_{2}, J_{2}\right)-S\left(M_{1}, J_{1}\right)$. This can be explicitly written as

$$
\begin{aligned}
\Delta S= & \frac{2 \pi^{2}}{9}\left[\sqrt{96 M_{2}^{3}-81 J_{2}^{2}}-\sqrt{96 M_{1}^{3}-81 J_{1}^{2}}\right] \\
& +\eta\left[\exp \left(-\frac{2 \pi^{2} \sqrt{96 M_{2}^{3}-81 J_{2}^{2}}}{9}\right)-\exp \left(-\frac{2 \pi^{2} \sqrt{96 M_{1}^{3}-81 J_{1}^{2}}}{9}\right)\right] .
\end{aligned}
$$

This change in the entropy can be used to obtain a change in the internal energy of this Myers-Perry black hole. This can be done by first noting that the internal energy of quantum Myers-Perry black holes (where we have neglected $\mathcal{O}\left(J^{4}\right)$ terms), can be expressed as

$$
\begin{aligned}
U \approx & \frac{-128 M^{4}+297 M J^{2}}{-128 M^{3}+378 J^{2}} \\
& -\frac{\eta}{28} \frac{-1792 \pi^{2} M^{6}+4158 \pi^{2} J^{2} M^{3}+243 \sqrt{6} J^{2} M^{\frac{3}{2}}}{\pi^{2} M^{2}\left(-64 M^{3}+189 J^{2}\right)} .
\end{aligned}
$$


Then, we can define the change in internal energy as $\Delta U=U\left(M_{2}, J_{2}\right)-U\left(M_{1}, J_{1}\right)$. So, we can express such a change in the internal energy of a Myers-Perry black hole evaporates as

$$
\begin{aligned}
\Delta U= & \frac{-128 M_{2}^{4}+297 M_{2} J_{2}^{2}}{-128 M_{2}^{3}+378 J_{2}^{2}}-\frac{-128 M_{1}^{4}+297 M_{1} J_{1}^{2}}{-128 M_{1}^{3}+378 J_{1}^{2}} \\
& -\frac{\eta}{28} \frac{-1792 \pi^{2} M_{2}^{6}+4158 \pi^{2} J_{2}^{2} M_{2}^{3}+243 \sqrt{6} J_{2}^{2} M_{2}^{\frac{3}{2}}}{\pi^{2} M_{2}^{2}\left(-64 M_{2}^{3}+189 J_{2}^{2}\right)} \\
& +\frac{\eta}{28} \frac{-1792 \pi^{2} M_{1}^{6}+4158 \pi^{2} J_{1}^{2} M_{1}^{3}+243 \sqrt{6} J_{1}^{2} M_{1}^{\frac{3}{2}}}{\pi^{2} M_{1}^{2}\left(-64 M_{1}^{3}+189 J_{1}^{2}\right)} .
\end{aligned}
$$

This quantity does not vanish, and the internal energy of the Myers-Perry black hole changes, as it evaporates from $\Omega\left(M_{2}, J_{2}\right)$ to $\Omega\left(M_{1}, J_{1}\right)$. Now it is important to analyze the amount of quantum work done as the internal energy of the Myers-Perry black hole changes from $U\left(M_{2}, J_{2}\right)$ to $U\left(M_{1}, J_{1}\right)$. This is because apart from Hawking radiation, a part of the internal energy of the black hole does quantum work during its evaporation. It may be noted that for equilibrium processes, where we can neglect change in the area of the black hole, we can also neglect this quantum work term. However, for quantum sized black holes, the change in the area is of the same order as the area, and we cannot neglect the quantum work done as the black hole evaporates. So, change in the total energy of the black hole is given by the energy radiated by the Hawking radiation, and the energy spend in doing quantum work $W$, as the black hole contracts. Now let the total amount of heat in Hawking radiation be denoted by $Q$, as the black hole evaporates from $\Omega\left(M_{2}, J_{2}\right)$ to $\Omega\left(M_{1}, J_{1}\right)$, then we can write [43]

$$
\Delta U=Q-\langle W\rangle
$$

where $\langle W\rangle$ is the average quantum work done as the black hole evaporates from $\Omega\left(M_{2}, J_{2}\right)$ to $\Omega\left(M_{1}, J_{1}\right)$. We can use the Jarzynski equality to obtain this quantum work done as the Myers-Perry black hole evaporates from $\Omega\left(M_{2}, J_{2}\right)$ to $\Omega\left(M_{1}, J_{1}\right)$ [64, 65]. Thus, using the Jarzynski equality $[66,67]$, we can write the quantum work in terms of difference of the equilibrium free energies of these two states $[68,69]$

$$
\left\langle e^{-\beta W}\right\rangle=e^{\beta \Delta F}
$$

So, it is possible to relate the quantum work done by the Myers-Perry black hole to the difference of free energies $F\left(M_{2}, J_{2}\right)$ and $F\left(M_{1}, J_{1}\right)$. It is important to note that unlike heat (represented by Hawking radiation), the quantum work done on a system is represented by a unitary information preserving process $[44,45]$. Thus, there is an unitary information preserving process associated with the evaporation of black holes. This unitary information preserving process, which corresponds to quantum work, can be neglected at large scales, but cannot be neglected at quantum scales. It could be possible that information can leak out of a black hole due to such a process. So, it is possible that the black hole information loss paradox $[46,47]$ occur only due to the use of equilibrium thermodynamics to describe the evaporation of a black hole down to Planck scale. However, at such a small 
scale, we have to analyze the system using non-equilibrium quantum thermodynamics. As quantum work, which is represented by an unitary information preserving process, becomes important at that scale, it is possible that black hole information paradox can be resolved by the use of non-equilibrium quantum thermodynamics. It would be interesting to use the relation between quantum information theory and non-equilibrium quantum thermodynamics to investigate the black hole information paradox [48, 49]. This can be done by using the results obtained in this paper, along with the relation between quantum work and information theory $[48,49]$. The presence of this unitary information preserving process might be the reason that the black hole information paradox does not occur in gauge gravity duality $[50,51]$. So, it would be interesting to explicitly analyze the holographic dual to quantum work, and its consequences on black hole information paradox. However, here we will only analyze the effect of quantum gravitational corrections on quantum work for a quantum sized Myers-Perry black hole.

Now, we can obtain the quantum work corrected by non-perturbative corrections using quantum corrected free energy. We can write this modified free energy, which has been corrected by non-perturbative quantum gravitational corrections as

$$
F=\frac{M}{3}+\frac{9}{16} \frac{J^{2}}{M^{2}}+\frac{\eta}{2 \pi^{2}} \int\left(\frac{\sqrt{96 M^{3}-81 J^{2}}}{8 M^{3}}-\frac{9}{\sqrt{96 M^{3}-81 J^{2}}}\right) e^{-\frac{2 \pi^{2} \sqrt{96 M^{3}-81 J^{2}}}{9}} d M .
$$

Now, for a quantum scale Myers-Perry black hole, we can approximate (neglecting $\mathcal{O}\left(J^{4}\right)$ ) the free energy as

$$
F \approx \frac{128 M^{3}+135 J^{2}}{384 M^{2}}-\frac{\eta}{2688 M^{\frac{7}{2}} \pi^{2}}\left[896 M^{\frac{7}{2}} \pi^{2}+945 M^{\frac{3}{2}} \pi^{2} J^{2}+336 \sqrt{6} M^{3}-81 \sqrt{6} J^{2}\right] .
$$

So, as the Myers-Perry black hole with microstates $\Omega\left(M_{1}, J_{1}\right)$ evaporates to microstates $\Omega\left(M_{2}, J_{2}\right)$, the change of free energy can be expressed as $\Delta F=F\left(M_{2}, J_{2}\right)-F\left(M_{1}, J_{1}\right)$. Thus, using Jarzynski equality, we can write the quantum work as

$$
\begin{aligned}
\left\langle e^{-\beta W}\right\rangle= & e^{\beta \Delta F} \\
= & \exp \beta\left[\frac{128 M_{2}^{3}+135 J_{2}^{2}}{384 M_{2}^{2}}-\frac{128 M_{1}^{3}+135 J_{1}^{2}}{384 M_{1}^{2}}\right. \\
& -\frac{\eta}{2688 M_{2}^{\frac{7}{2}} \pi^{2}}\left[896 M_{2}^{\frac{7}{2}} \pi^{2}+945 M_{2}^{\frac{3}{2}} \pi^{2} J_{2}^{2}+336 \sqrt{6} M_{2}^{3}-81 \sqrt{6} J_{2}^{2}\right] \\
& \left.+\frac{\eta}{2688 M_{1}^{\frac{7}{2}} \pi^{2}}\left[896 M_{1}^{\frac{7}{2}} \pi^{2}+945 M_{1}^{\frac{3}{2}} \pi^{2} J_{1}^{2}+336 \sqrt{6} M_{1}^{3}-81 \sqrt{6} J_{1}^{2}\right]\right] .
\end{aligned}
$$

Thus, we have obtained quantum work for a Myers-Perry black hole. We observe that it depends on the strength of non-perturbative corrections.

We can write the partition function for a Myers-Perry black using its microstates [58]. So, as the microstates change from $\Omega\left(M_{1}, J_{1}\right)$ to $\Omega\left(M_{2}, J_{2}\right)$ during evaporation, the partition function changes from $Z_{1}\left[\Omega\left(M_{1}, J_{1}\right)\right]$ to $Z_{2}\left[\Omega\left(M_{1}, J_{2}\right)\right]$. We can relate these partition 

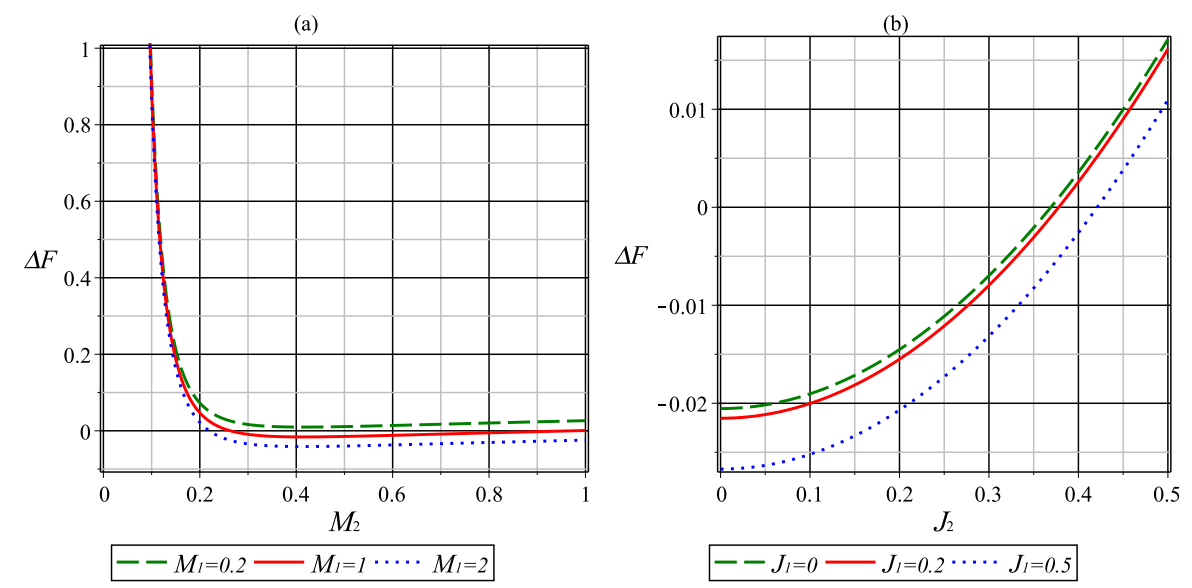

Figure 2. $\Delta F$ of the Myers-Perry black hole in terms of (a) final mass with $\eta=0.95, J_{1}=0.1$, and $J_{2}=0.2$; (b) final angular momentum with $\eta=0.95, M_{1}=0.5$, and $M_{2}=1$.

functions for a Myers-Perry black hole to the quantum work done as the black hole evaporates between these two microstates. So, if the black hole with a partition function $Z_{1}$ evaporates to a black hole with a partition function $Z_{2}$, then we can use the Jarzynski equality to obtain $[66,67]$

$$
\left\langle e^{-\beta W}\right\rangle=\frac{Z_{2}}{Z_{1}}
$$

As $Z_{2} / Z_{1}$ has been expressed in terms quantum work, we can also express the relative weights of the partition function in terms of the difference between the equilibrium free energies as $\exp (\beta \Delta F)=Z_{2} / Z_{1}$.

Now, we can use Jensen inequality to relate the average of exponential of quantum work to exponential of the average of quantum work for a Myers-Perry black hole as $e^{\langle-\beta W\rangle} \leq\left\langle e^{-\beta W}\right\rangle$. So, by using this inequality we can obtain an inequality for the quantum work done during the evaporation of a Myers-Perry black hole. This can be written in terms of the difference of the free energy, which in term can be written in terms of the masses $M_{1}, M_{2}$ and angular momentum $J_{1}, J_{2}$ as

$$
\begin{aligned}
\langle W\rangle \geq & \Delta F \\
= & \frac{128 M_{2}^{3}+135 J_{2}^{2}}{384 M_{2}^{2}}-\frac{128 M_{1}^{3}+135 J_{1}^{2}}{384 M_{1}^{2}} \\
& -\frac{\eta}{2688 M_{2}^{\frac{7}{2}} \pi^{2}}\left[896 M_{2}^{\frac{7}{2}} \pi^{2}+945 M_{2}^{\frac{3}{2}} \pi^{2} J_{2}^{2}+336 \sqrt{6} M_{2}^{3}-81 \sqrt{6} J_{2}^{2}\right] \\
& +\frac{\eta}{2688 M_{1}^{\frac{7}{2}} \pi^{2}}\left[896 M_{1}^{\frac{7}{2}} \pi^{2}+945 M_{1}^{\frac{3}{2}} \pi^{2} J_{1}^{2}+336 \sqrt{6} M_{1}^{3}-81 \sqrt{6} J_{1}^{2}\right] .
\end{aligned}
$$

Here, $\langle W\rangle=\Delta F$ for processes at equilibrium. It may be noted that in figure 2 , we have analyzed the effect of non-perturbative corrections on the difference of free energy between two states of a Myers-Perry black hole. This was done by first analyzing the variation of the difference of free energy with mass, and then analyzing the variation of difference of 

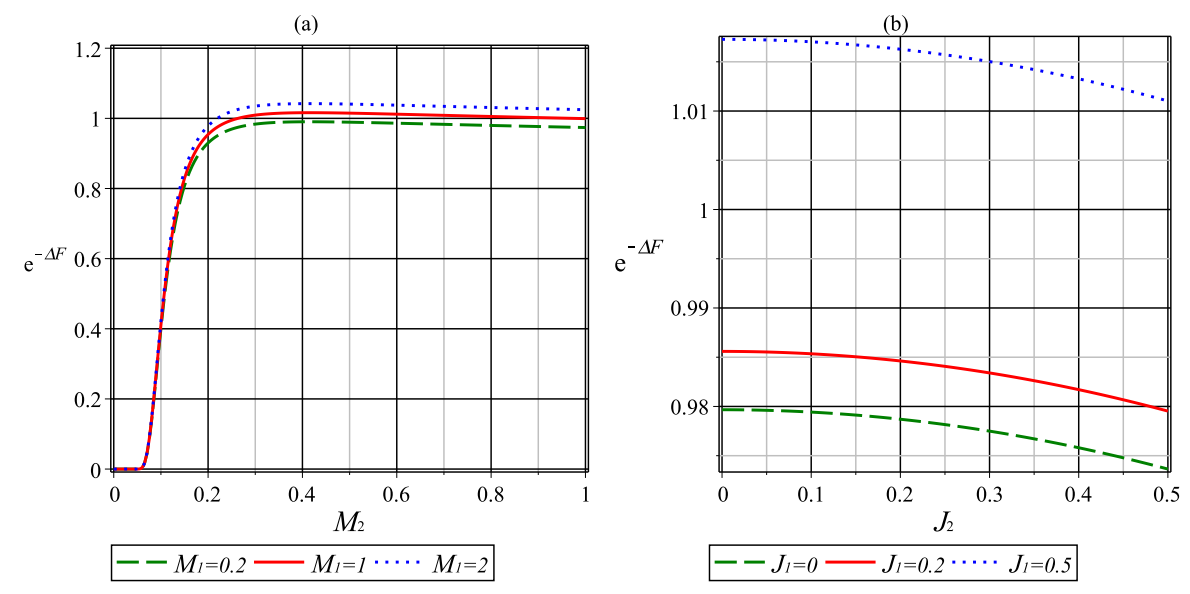

Figure 3. $e^{-\Delta F}=Z_{2} / Z_{1}$ of the Myers-Perry black hole in terms of in terms of (a) final mass with $\eta=0.95, J_{1}=0.1$, and $J_{2}=0.2$; (b) final angular momentum with $\eta=0.95, M_{1}=0.5$, and $M_{2}=1$.

free energy with angular momentum. As the quantum work is bounded in terms of the free energy, we can view this as the correction to the bound on the quantum work corrected by non-perturbative corrections.

In the figure 3, we will investigate the effects of non-perturbative corrections to the relative weights of the partition functions. As these relative weights can be expressed in terms of the difference of free energies, and this difference is corrected by non-perturbative corrections, these weights are also corrected by non-perturbative corrections. It may be noted that weights can also be related to the exponential of quantum work using the Jarzynski equality $[66,67]$. Here, again we investigate the variation of these relative weights with the difference of mass and difference of angular momentum of the Myers-Perry black hole. We can observe that non-perturbative quantum effects can produce interesting modifications to the relative weights of such partition functions.

As the quantum work is done during the evaporation of a Myers-Perry black hole, it is important to analyze the effects of these non-perturbative corrections to the stability of these quantum scale Myers-Perry black hole. This can be done by analyzing the nonperturbative corrections to the specific heat of a Myers-Perry black hole

$$
C=\frac{16 \pi^{2} M^{3} \sqrt{96 M^{3}-81 J^{2}}\left(1-\eta e^{-\frac{2 \pi^{2} \sqrt{96 M^{3}-81 J^{2}}}{9}}\right)}{81 J^{2}-24 M^{3}} .
$$

The information about the stability of this thermodynamics system can be obtained from the sign of this corrected specific heat. We plot the specific heat for the different values of $M$ in figure 4. We observe that the original Myers-Perry black hole with large value of $M$ is in an unstable phase. However, there is a minimum mass, at which specific heat vanish $C=0$. So, at this stage the black hole does not exchange energy with the surroundings. Furthermore, at this stage $T=0$, and the Myers-Perry black hole stops radiating Hawking radiation. So, at this stage it forms a stable Myers-Perry black remnant. 

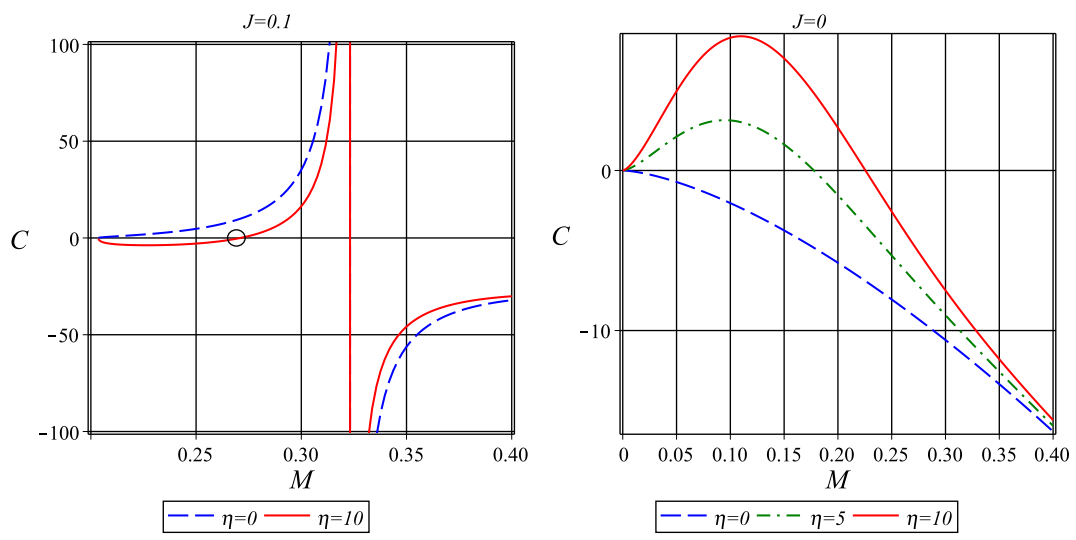

Figure 4. Specific heat of the Myers-Perry black hole in terms of mass.

The non-perturbative quantum corrections change this behavior, as can be observed from the left part of plot in figure 4. So, due to the non-perturbative quantum corrections, the specific heat of a quantum scale Myers-Perry black hole becomes negative, and it becomes unstable.

At large scales, the original Myers-Perry black hole in an unstable phase. At such a large scale, the effects from non-perturbative quantum corrections can be neglected. However, a first order phase transition occurs at a critical scale, for the original MyersPerry black hole. After this phase transition, the original Myers-Perry black is in a stable phase. The black hole mass at this critical point, where such a phase transition occurs is given by

$$
M_{c}=\left[\frac{81}{96}\left(J^{2}+\left(\frac{\ln \eta}{2 \pi^{2}}\right)^{2}\right)\right]^{1 / 3} .
$$

It is denoted by a circle line in left plot of figure 4 . However, at such a small quantum scales $\left(M \leq M_{c}\right)$, non-perturbative quantum corrections become important, and cannot be neglected. We observe that due to these non-perturbative quantum corrections, the Myers-Perry black hole becomes unstable, at such small scales. It is interesting to note that the situation is different, when the angular momentum is neglected, $J=0$. Here, the original black hole (Schwarzschild black hole) is initially unstable, and the non-perturbative corrections make it stable at small scales, as illustrated by right plot of figure 4 . Thus, we see that the behavior of quantum corrected specific heat depends on both $J$ and $M$. We also observe that the stability of the Myers-Perry black holes is non-trivially modified by the non-perturbative quantum corrections at small scales.

\section{Information geometry}

It is possible to investigate the stability of black holes using information geometry [70, 71]. The phase transitions for black holes can be investigated using divergences of the Ricci scalar of the information geometry [72-77]. This Ricci scalar is obtained using from a specific form of the information metric. There are different information theoretical metrics, 
which represent different amount of information about a thermodynamic system. In fact, it has been observed that the information about phase transition of different black holes can only be represented by certain informational theoretical metrics. Thus, it is important to use various information theoretical metrics to analyze the phase transition in a black hole. The phase transition in a charged Gauss-Bonnet AdS black holes has been studied using a Ruppiner metric [79], and the phase transition in a Park black hole has been studied using a Weinhold metric [80]. However, it has been demonstrated that both the Weinhold and Ruppiner metrics cannot be used to obtain information about the phase transition of a charged AdS black hole with a global monopole [78]. It is possible to obtain such information about the phase transition of such a charged AdS black hole with a global monopole from Quevedo and HPEM metrics [78]. The phase transition of a black hole surrounded by the perfect fluid in Rastall theory has been studied using the Ruppiner and HPEM metrics, and it has been observed such a phase transition cannot be analyzed using Weinhold and Quevedo metrics [81]. It has been demonstrated that for such a black hole surrounded by the perfect fluid in the Rastall theory, it is possible to extract more information from the HPEM metrics than the Ruppiner metric [81]. Thus, different amount of physical information about the phase transition can be extracted from different information theoretical metrics.

We have observed that at quantum scales, the quantum gravitational corrections cannot be neglected. These quantum gravitational corrections are expected to modify the information theoretical metrics for a black hole. So, we need to modify the original formalism of the information geometry to incorporate these quantum gravitational effects. As these information theoretical metrics are expressed in terms of the mass of a black hole, we need to define a novel quantum mass of a Myers-Perry black hole to construct effective quantum corrected information metrics. This can be done by directly incorporating the effect of non-perturbative quantum gravitational corrections into the quantum mass of a Myers-Perry black hole. Furthermore, as the quantum gravitational corrections can be neglected for a Myers-Perry black hole at large scales, this quantum mass should reduces to the original mass of the Myers-Perry black hole at such large scales.

Such a quantum mass for a Myers-Perry black hole can be defined by first observing that the original entropy (without quantum gravitational corrections) of the Myers-Perry black hole is a function of original mass and angular momentum [37, 38]. So, the original mass can be expressed as a function of the original entropy and angular momentum. Now as the quantum gravitational corrections modify the original entropy, the quantum gravitationally corrected entropy can be used to define this novel quantum mass for the Myers-Perry black hole. Thus, the quantum mass can be expressed as function of the quantum corrected entropy

$$
M(S, J)=\frac{3}{8 \pi^{4 / 3}}\left[16 \pi^{4} J^{2}+4 L W\left(-\frac{\eta}{e^{S}}\right)^{2}+8 S L W\left(-\frac{\eta}{e^{S}}\right)+4 S^{2}\right]^{\frac{1}{3}}
$$

where $L W(x)$ is Lambertw function. Here, we can consider the parameter $\eta$ as a new 
thermodynamic variable with conjugate $X$, which is given by,

$$
X=\frac{\sqrt{96 M^{3}-81 J^{2}}}{32 \pi^{2} M^{2}} e^{-\frac{2 \pi^{2} \sqrt{96 M^{3}-81 J^{2}}}{9}} .
$$

Now, we can obtain the non-perturbative corrections for other thermodynamic quantities as a function of $S$ and $J$. So, we can write the quantum gravitationally corrected temperature $(T=(\partial M / \partial S))$ as

$$
T=\frac{\xi_{1}}{8 \xi_{2}^{2 / 3} \pi^{4 / 3}},
$$

We can also express the quantum gravitationally corrected specific heat $(C=T(\partial S / \partial T))$ as

$$
C=\frac{\xi_{1}}{8 \xi_{2}{ }^{2 / 3} \pi^{4 / 3}}\left(-\frac{\xi_{1}^{2}}{12 g^{5 / 3} \pi^{4 / 3}}+\frac{\xi_{3}}{8 g^{2 / 3} \pi^{4 / 3}}\right)^{-1}
$$

where

$$
\begin{aligned}
\xi_{1}= & -8 \frac{\left(L W\left(-\eta \mathrm{e}^{-S}\right)\right)^{2}}{1+L W\left(-\eta \mathrm{e}^{-S}\right)}+8 L W\left(\eta \mathrm{e}^{-S}\right)-8 \frac{S L W\left(\eta \mathrm{e}^{-S}\right)}{1+L W\left(\eta \mathrm{e}^{-S}\right)}+8 S \\
\xi_{2}= & 16 \pi^{4} J^{2}+4\left(L W\left(-\eta \mathrm{e}^{-S}\right)\right)^{2}+8 S L W\left(\eta \mathrm{e}^{-S}\right)+4 S^{2} \\
\xi_{3}= & -16 \frac{L W\left(\eta \mathrm{e}^{-S}\right)}{1+L W\left(\eta \mathrm{e}^{-S}\right)}+\frac{8 S L W\left(\eta \mathrm{e}^{-S}\right)+16\left(L W\left(-\eta \mathrm{e}^{-S}\right)\right)^{2}}{\left(1+L W\left(-\eta \mathrm{e}^{-S}\right)\right)^{2}} \\
- & \frac{8\left(L W\left(-\eta \mathrm{e}^{-S}\right)\right)^{3}+8 S L W\left(\eta \mathrm{e}^{-S}\right)}{\left(1+L W\left(-\eta \mathrm{e}^{-S}\right)\right)^{3}}+8 .
\end{aligned}
$$

We have plotted the behavior of these thermodynamic quantities and investigated the effects of non-perturbative gravitational quantum corrections on them. Now from figure 5, figure 6 , figure 7 and we observe that the quantum corrected mass, temperature and specific heat depends on $\eta$ and $J$. This is expected as $\eta$ is the parameter which measures the strength of non-perturbative quantum corrections. Also, the angular momentum can change the effect of the non-perturbative quantum corrections on the thermodynamics of a Myers-Perry black hole. Here it has been demonstrated how the quantum gravitational corrections are first directly incorporated in the quantum mass, and then their effect on the angular momentum is obtain from this quantum mass. This analysis can be generalized for charged black holes, and other black hole solutions with different topology. As the information geometry of a black hole could be expressed using its mass, we can use the quantum mass to study the quantum gravitational corrections to the information geometry for a quantum Myers-Perry black hole. This will be done by analyzing the quantum gravitational corrections to different information theoretical metrics [82-88, 92, 93] of a quantum Myers-Perry black hole. We use different informational theoretical metrics to ensure that we do not lose important physical information about the effects of the non-perturbative quantum gravitational corrections on phase transitions.

We will start this analysis using the Ruppeiner metric. The Ruppeiner metric was proposed to define a geometry for the space of thermodynamical fluctuations [82, 83]. Now 


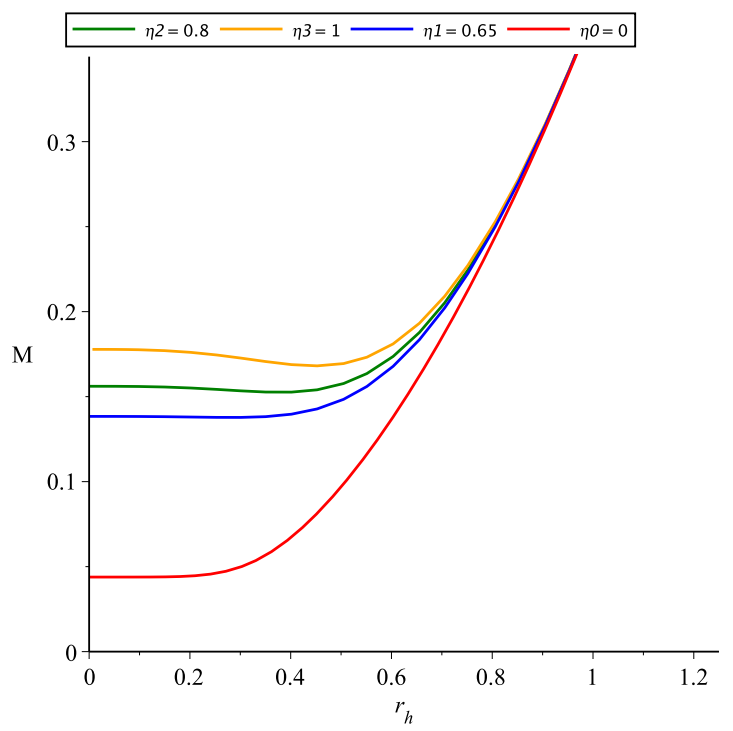

(a) $J=0.01$

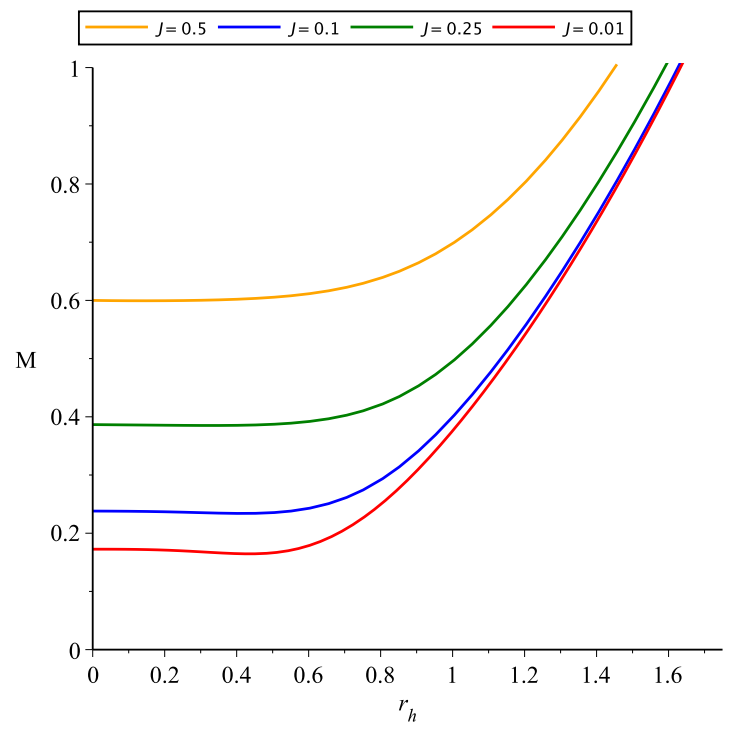

(b) $\eta=0.95$

Figure 5. Variations of mass in terms of horizon radius $r_{h}$, for the Myers-Perry black hole in five dimensions.

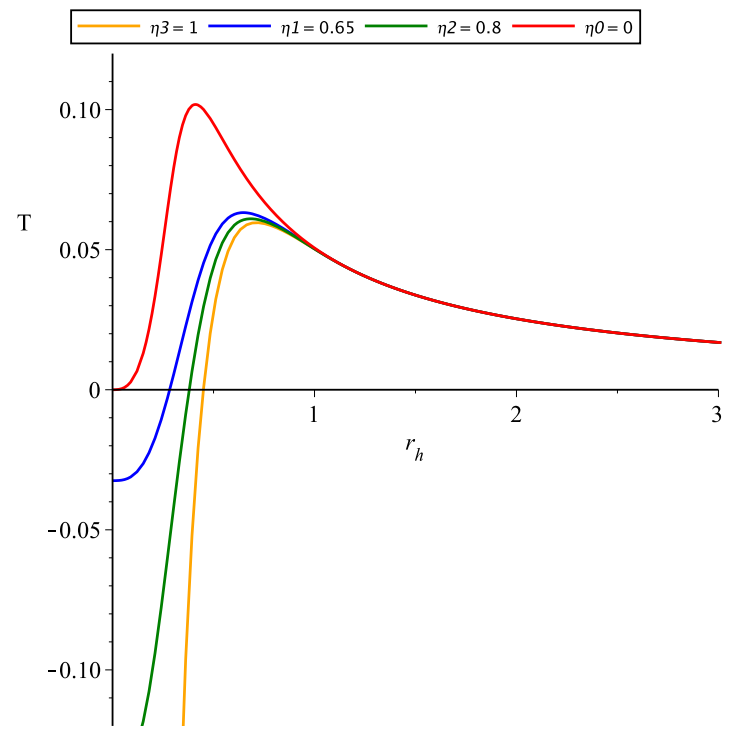

(a) $J=0.01$

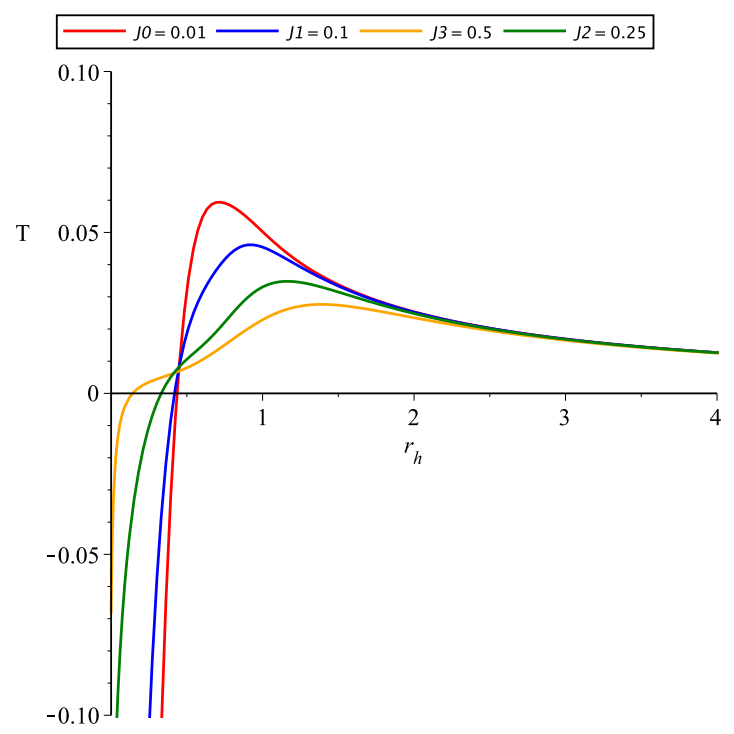

(b) $\eta=0.95$

Figure 6. Variations of temperature in terms of horizon radius $r_{h}$, for the Myers-Perry black hole in five dimensions. 


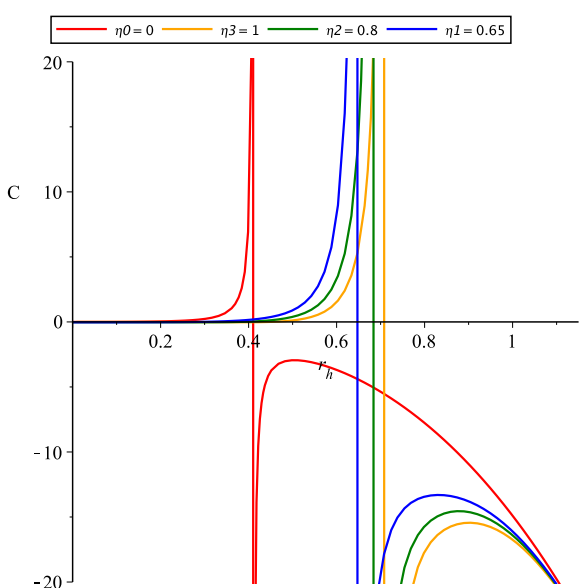

(a) $J=0.01$

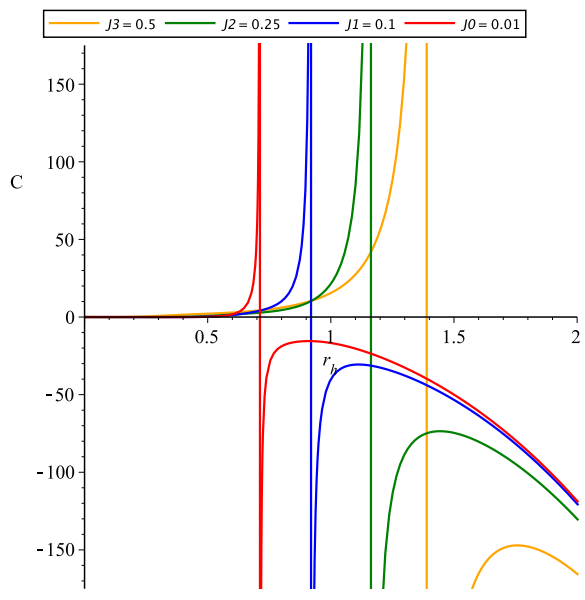

(c) $\eta=0.95$

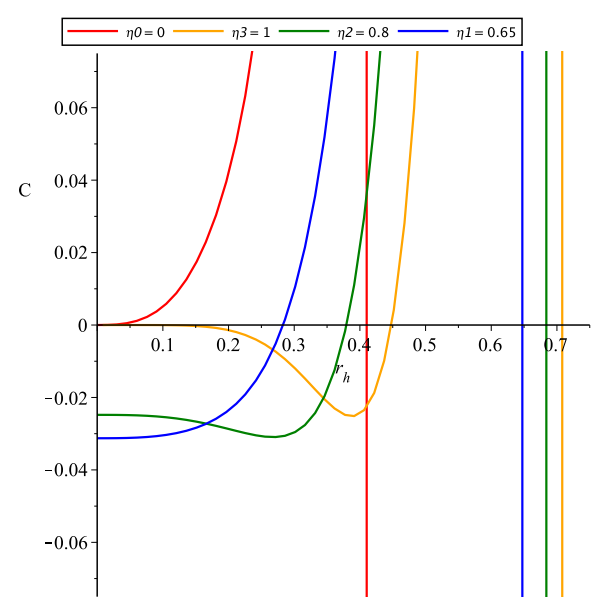

(b) closeup of figure (a)

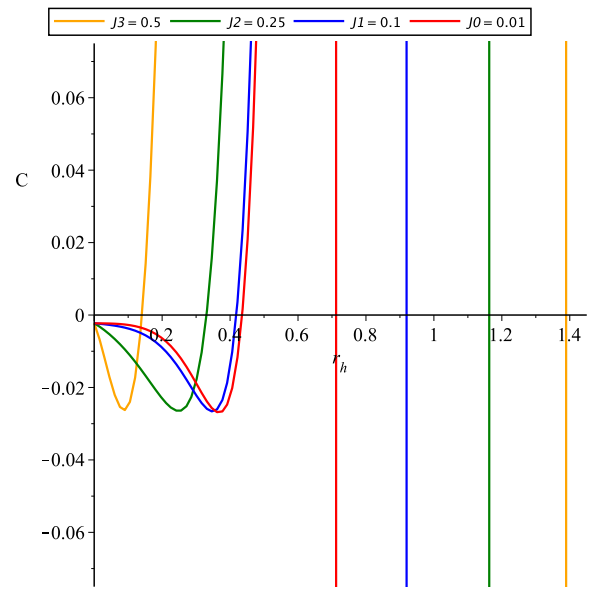

(d) closeup of figure (c)

Figure 7. Variations of the specific heat in terms of horizon radius $r_{h}$, for the Myers-Perry black hole in five dimensions.

we can define the quantum corrected effective Ruppeiner metric as

$$
d s^{2}=-\frac{1}{T} M g_{a b}^{R} d X^{a} d X^{b}
$$

The Weinhold metric was motivated using the connections between Gibbs-Duhem relation and scaling of thermodynamic potentials $[84,85]$. So, we can write the quantum corrected effective Weinhold metric as

$$
d s^{2}=M g_{a b}^{W} d X^{a} d X^{b}
$$

The Quevedo (I and II) metrics are constructed using Legendre invariant set of metrics in the phase space $[86,87]$. It may be noted that their pullback produces the required information metrics on the space of equilibrium states. Now motivated by the original Quevedo (I and II) metrics, we can define the quantum corrected effective Quevedo (I and II) 
metrics as

$$
d s^{2}=\left(S M_{S}+\alpha M_{\alpha}\right)\left(-M_{S S} d S^{2}+M_{\alpha \alpha} d \alpha^{2}\right),
$$

and

$$
d s^{2}=S M_{S}\left(-M_{S S} d S^{2}+M_{\alpha \alpha} d \alpha^{2}\right) .
$$

The HPEM information metric has been constructed by using a different conformal than the Quevedo metrics [88-91]. Thus, the quantum corrected effective HPEM metrics can be expressed as

$$
d s^{2}=\frac{S M_{S}}{\left(\frac{\partial^{2} M}{\partial \alpha^{2}}\right)^{3}}\left(-M_{S S} d S^{2}+M_{\alpha \alpha} d \alpha^{2}\right) .
$$

It is also possible to define NTG metric by changing coordinates of the thermodynamic space by Jacobean transformation $[92,93]$. Now the quantum corrected effective NTG metric can be written as

$$
d s^{2}=\frac{1}{T}\left(-M_{S S} d S^{2}+M_{\alpha \alpha} d \alpha^{2}\right) .
$$

In these quantum corrected effective metrics, $M_{X Y}$ is the second order differentiation with respect to $X$ and $Y$ variables. It may be noted that they have been obtained using the quantum corrected mass.

Now, we can use plots to investigate the behavior of quantum corrected effective Ricci scalars, and compare it with the behavior of the specific heat (see figures 8, 9). We find that quantum corrected effective Weinhold metric does not have any divergences, where the specific heat diverges or becomes zero (see figures 8(a)). Furthermore, from figure 8(b), we observe that the quantum corrected effective Ruppeiner metric has a divergence, where the specific heat becomes zero. So, the divergence of the scalar curvature for the quantum corrected effective Ruppeiner metric corresponds to a phase transition in this system. The situation is different for the quantum corrected effective Quevedo (I, II), quantum corrected effective HPEM, and quantum corrected effective NTG metrics. The divergences of the scalar curvature of these quantum corrected effective metrics can provide better information about the phase transition points for a Myers-Perry black hole (see figures 8(c), (d) and 9(a)-(d)). In fact, we observe that the divergent points of the quantum corrected effective Ricci scalar for the quantum corrected effective Quevedo (I, II), quantum corrected effective HPEM and quantum corrected effective NTG metrics contain important information about phase transitions type in this system. The divergent points of the quantum corrected effective Ricci scalar for these quantum corrected effective metrics coincide with both zero and divergent points of the specific heat. So, these quantum corrected effective metrics contain more information about the phase transition in this system. Thus, quantum corrected effective Quevedo (I, II), quantum corrected effective HPEM and quantum corrected effective NTG metrics can be used to obtain important physical information about the phase transition in a quantum Myers-Perry black hole. Here we observe that different quantum corrected informational metrics also contain different amounts of information about the system. It is interesting to note that the information geometries have 


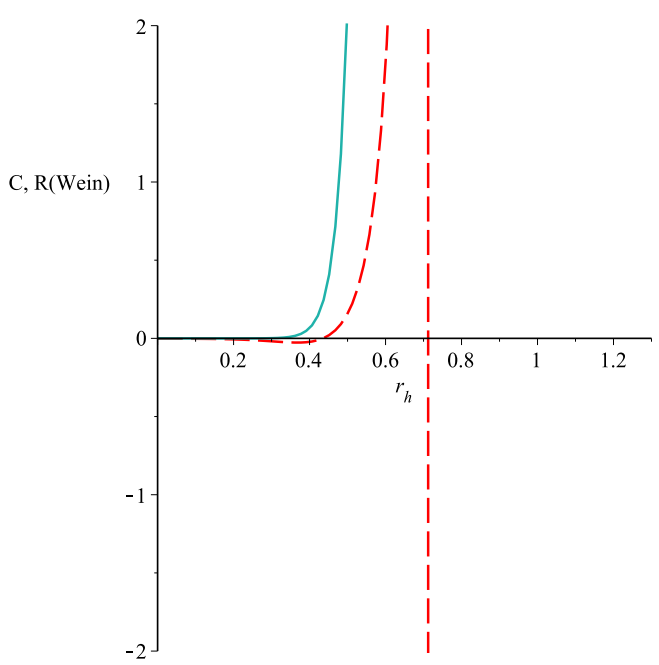

(a)

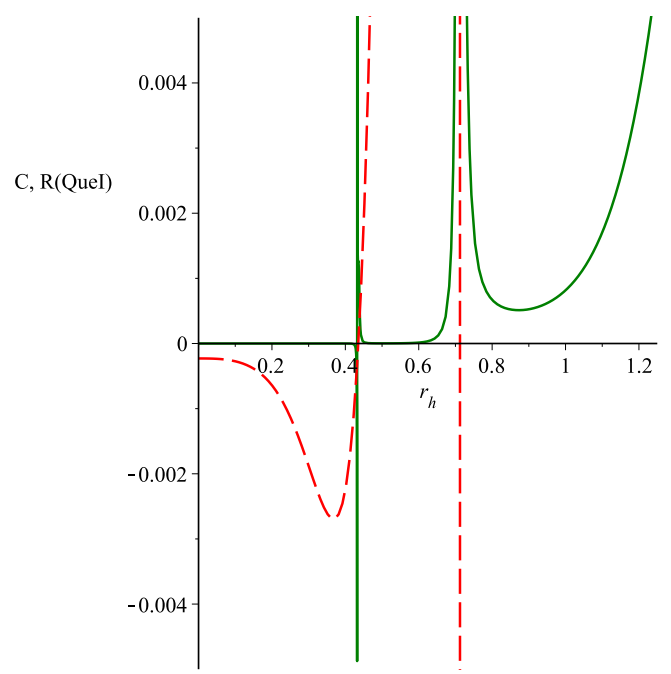

(c)

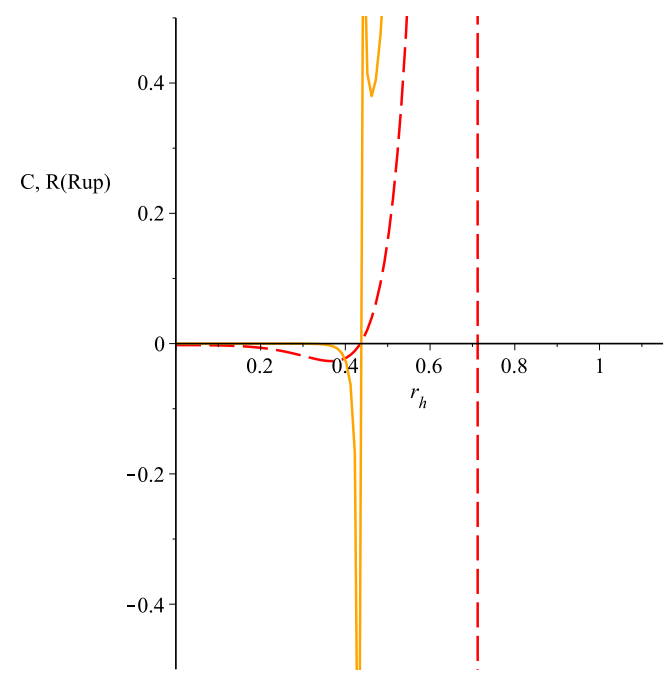

(b)

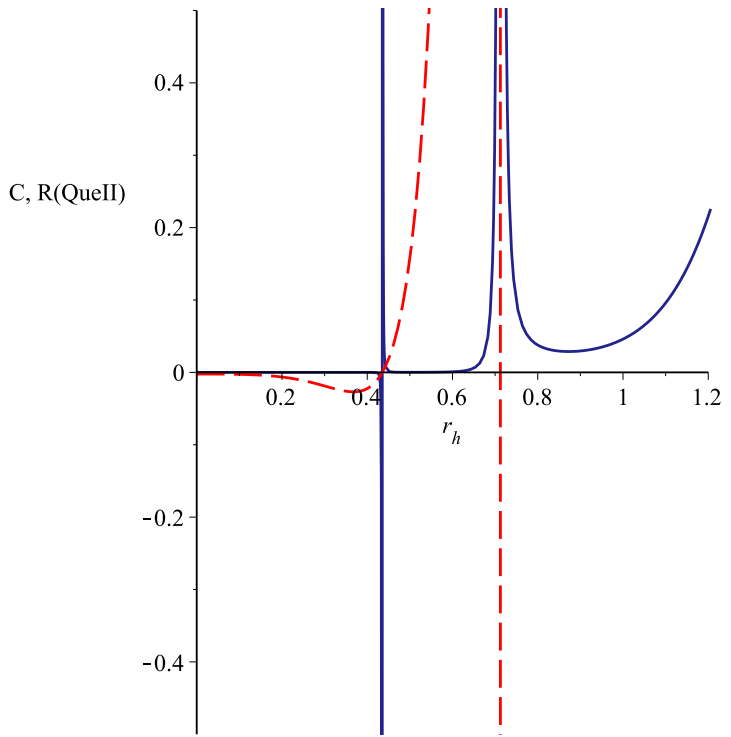

(d)

Figure 8. Curvature scalar variation of Weinhold (light green line), Ruppeiner (orange line), Quevedo case I (green line) and Quevedo case II (navy line) metrics and also specific heat (red dash line), in terms of horizon radius $r_{h}$ for $\eta=0.95$ and $J=0.01$, for a Myers-Perry black hole. 


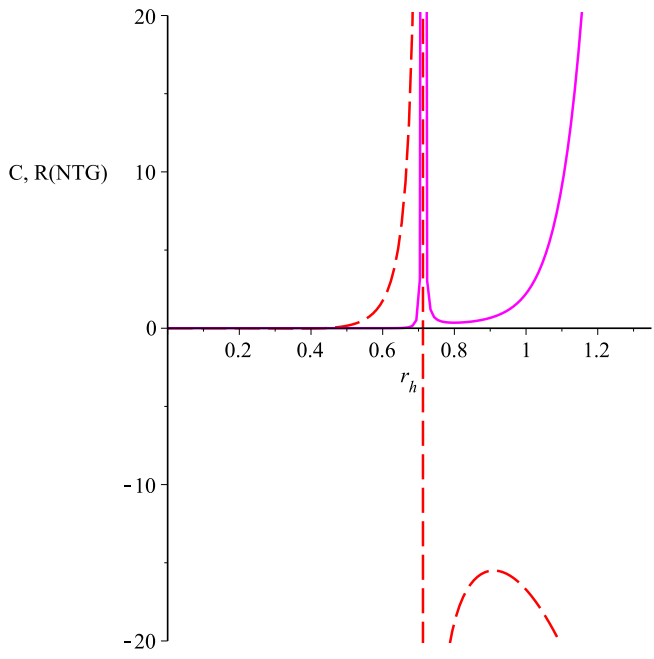

(a)

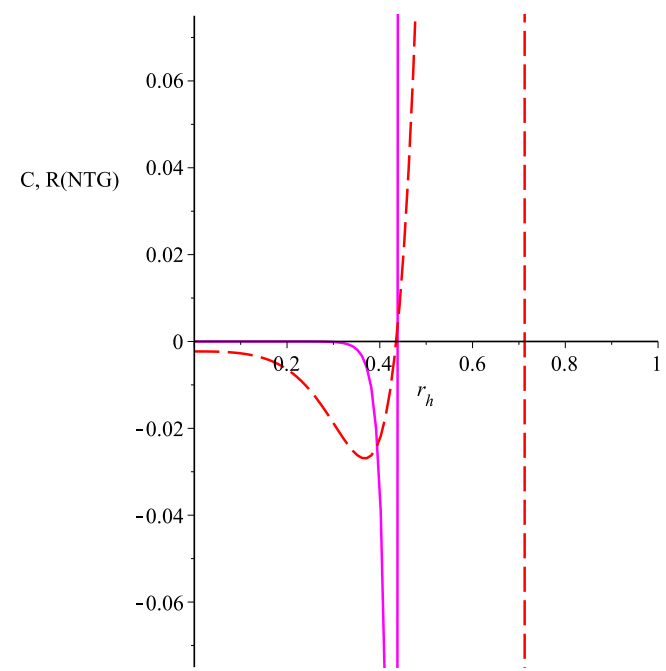

(b) closeup of figure (a)

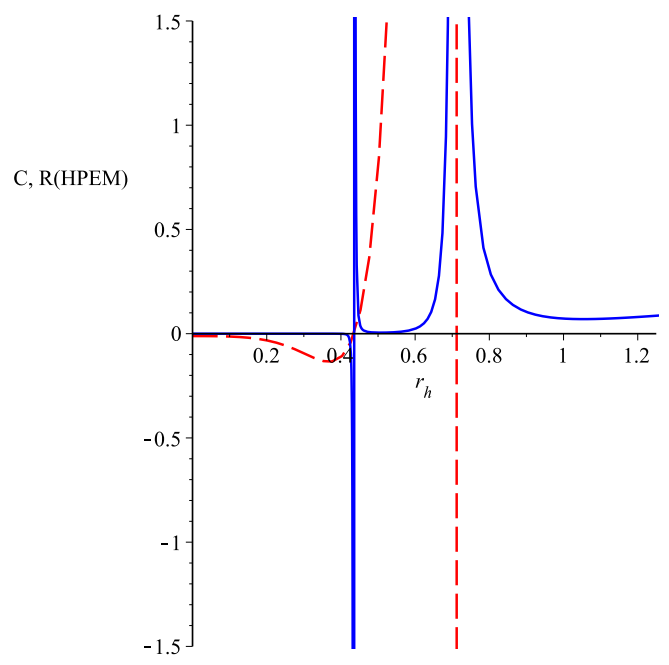

(c)

Figure 9. Scalar curvature of NTG (magenta line) and HPEM (blue line) metrics and also specific heat (red dash line), in terms of horizon radius $r_{h}$ for $\eta=0.95$ and $J=0.01$, for a Myers-Perry black hole.

been constructed by incorporating quantum gravitational corrections in the effective informational metrics, which were obtained from a novel quantum mass of a Myers-Perry black hole.

\section{Conclusion}

In this paper, we analyzed quantum work for a quantum scale Myers-Perry black hole. It was observed that the quantum work was corrected by non-perturbative correction for a quantum scale Myers-Perry black hole. As these corrections changed the relation between 
entropy and area of a Myers-Perry black hole, they also produces corrections in free energy of a Myers-Perry black hole. So, the difference of corrected free energy was used to obtain the corrections to the quantum work. We used the Jarzynski equality to obtain the corrected quantum work for this black hole.

It may be noted that it is possible to use the original entropy $S_{0}$ of the black hole to obtain quantum work. As the original entropy $S_{0}=A / 4$ is obtained by semi-classical approximations (using quantum field theory in curved space-time), the equilibrium thermodynamic quantities obtained from $S_{0}$ correspond to this approximation $[1,2]$. Now as the difference between such equilibrium free energies can be related to quantum work using the Jarzynski equality, the Jarzynski equality has been used to obtain the work corresponding to the original entropy $S_{0}$ of black holes $[68,69]$. However, for quantum scale black holes, it has been argued that non-perturbative quantum gravitational corrections will correct this original entropy [32-35]. These non-perturbative quantum gravitational corrections produce corrections to the original semi-classical entropy $S_{0}$, which can be represented as $S=S_{0}+\eta \exp \left(-S_{0}\right)$ [32-35]. Now it is possible to obtain the quantum gravitational corrections to the free energies from this quantum corrected entropy, we obtain quantum gravitational corrections to the quantum work for a Myers-Perry black hole. These quantum gravitational corrections are controlled by a parameter, and the behavior of the quantum work explicitly depended on this control parameter. It is possible to use the mircostates of a black hole to construct its partition function. As the black hole evaporates, its microstates change, and this changes the partition function of the system. We obtained the relative weights of such partitions functions using the Jarzynski equality for a quantum Myers-Perry black hole. Unlike heat represented by Hawking radiation, this quantum work is represented by a unitary information preserving process. So, there is an unitary information preserving process associated with the evaporation of black holes, and this can have implication for black hole information paradox. As quantum work was done during the evaporation of a Myers-Perry black hole, we also investigated the effect of such corrections on the stability of a Myers-Perry black hole. It was observed that even though the original Myers-Perry black hole become stable at short distance, the non-perturbative quantum corrections changed this behavior. These corrections depended on the angular momentum of the black hole. So, in absence of angular momentum, these corrections stabilized the quantum scale Myers-Perry black hole. We analyzed the quantum corrected information geometry of these black holes with different quantum corrected effective information metrics. It was observed that quantum corrected effective Quevedo (I, II), quantum corrected effective HPEM and quantum corrected effective NTG metrics contain more information about the phase transition in this system, than the quantum corrected effective Weinhold and quantum corrected effective Ruppeiner metrics. These results obtained by incorporating quantum gravitational corrections in effective informational metrics, which was obtained using a novel quantum mass of a Myers-Perry black hole.

It is known that the entropy of a back hole can be obtained from microstates of a conformal field theory. The microstates of black holes with angular momentum have also been studied using the AdS/CFT correspondence. Thus, it is possible to analyze the microstates of a rotating black hole using a conformal field theory dual to such a system. Furthermore, 
in this paper, we could derive the expression for work done during the evaporation of a Myers-Perry black hole. This was done using the Jarzynski equality for such a system. It is possible to investigate this using the AdS/CFT correspondence, and find the field theory dual to such work done during the evaporation of a black hole. We could analyze the quantum corrected information geometry of black holes using the AdS/CFT correspondence. It is expected that these different information theoretical metrics would be produce interesting dual structures for a Myers-Perry black hole. It is possible to extend this work to other black holes. These are solutions for an extended objects surrounded by event horizons. It is known that these exotic solutions still have the unusual causal structure. It is also possible to relate the black strings to fundamental strings of the string theory. It has been known that these black strings can have the usual properties associated with black holes. Thus, it is possible to analyze the thermodynamics of black strings, and even use the information theoretical metrics to analyze phase transitions in black strings. So, it would be interesting to investigate the effect of these non-perturbative quantum corrections on the thermodynamics of black strings. It is possible to investigate this modified thermodynamics of black strings using different information theoretical metrics. We can also obtain the quantum work for black stings using the Jarzynski equality for such a system. We could explicitly obtain the quantum work for black strings using the difference of their free energies.

It is known that in the Jacobson formalism, space-time emerges from the thermodynamics of the system. Now, it has been observed that the thermodynamics is corrected due to non-perturbative quantum corrections. So, it is possible to use this quantum corrected thermodynamics for the Myers-Perry black hole to obtain a quantum corrected effective metric for such black holes. These quantum corrections in this effective metric are expected be proportional to the coefficient of the non-perturbative corrections. Thus, at large distances, this quantum corrected geometry of a Myers-Perry black hole could be approximated by its classical metric. However, the quantum corrections to the metric would become important at short distances. It would be interesting to investigate the Raychaudhuri equation for such quantum gravitationally corrected effective geometries. This could then be used to investigate the effect of quantum corrections on the singularity theorems. It is expected that the geometry flow would also be modified by such non-perturbative quantum corrections. So, it would also be interesting to investigate what such effects for a Myers-Perry black hole, when its thermodynamics is modified from non-perturbative quantum corrections.

As we analyzed the effect of quantum gravitational corrections on a Myers-Perry black hole, it would be also interesting to analyze quantum work for fuzzballs. It is possible to analyze the black holes in string theory with fuzzball proposal. In this proposal, the singularity at the center of a black hole is smoothed out. This is done by assuming that the entire region inside the horizon of a black hole is made of string states, and these string states resemble a black hole. As the effective classical description of a fuzzball still resembles a black hole, it is consistent with large scale gravitational experiments conducted on large scale astrophysical black holes. The large scale thermodynamics from fuzzballs also resembles classical black holes. However, we do expect that short distances physics of fuzzballs to be different from black holes. So, it would be interesting to investigate 
the effect of quantum corrections on fuzzballs. It would also be possible to obtain the quantum work for fuzzballs by analyzing quantum thermodynamics of fuzzballs. It is also expected that the quantum work of fuzzballs would be corrected from short distance quantum corrections. It is possible to investigate such corrections to quantum work for fuzzballs using the Jarzynski equality. Thus, we can first try to obtain the equilibrium thermodynamic quantities for a fuzzball. The difference of two equilibrium free energies at two different states can then be related to the average quantum work. In fact, this average quantum work is related to the different weights of the partition functions, and the partition functions can be calculated in a fuzzballs in terms of string microstates. So, it would be interesting to related such partition functions using quantum work for a fuzzball.

Open Access. This article is distributed under the terms of the Creative Commons Attribution License (CC-BY 4.0), which permits any use, distribution and reproduction in any medium, provided the original author(s) and source are credited.

\section{References}

[1] S.W. Hawking, Black hole explosions, Nature 248 (1974) 30 [INSPIRE].

[2] S.W. Hawking, Particle Creation by Black Holes, Commun. Math. Phys. 43 (1975) 199 [Erratum ibid. 46 (1976) 206] [INSPIRE].

[3] L. Susskind, The world as a hologram, J. Math. Phys. 36 (1995) 6377 [hep-th/9409089] [INSPIRE].

[4] R. Bousso, The holographic principle, Rev. Mod. Phys. 74 (2002) 825 [hep-th/0203101] [INSPIRE].

[5] G. Lifschytz and M. Ortiz, Black hole thermodynamics from quantum gravity, Nucl. Phys. B 486 (1997) 131 [hep-th/9510115] [INSPIRE].

[6] S. Mahapatra, Logarithmic black hole entropy corrections and holographic Rényi entropy, Eur. Phys. J. C 78 (2018) 23 [arXiv: 1609.02850] [InSPIRE].

[7] K. Nozari and A.S. Sefidgar, On the existence of the logarithmic correction term in black hole entropy-area relation, General Relativity and Gravitation 39 (2007) 501.

[8] C. Keeler, F. Larsen and P. Lisbão, Logarithmic Corrections to $N \geq 2$ Black Hole Entropy, Phys. Rev. D 90 (2014) 043011 [arXiv: 1404.1379] [InSPIRE].

[9] D. Bak and S.-J. Rey, Holographic principle and string cosmology, Class. Quant. Grav. 17 (2000) L1 [hep-th/9811008] [INSPIRE].

[10] S. Kalyana Rama, Holographic principle in the closed universe: A resolution with negative pressure matter, Phys. Lett. B 457 (1999) 268 [hep-th/9904110] [InSPIRE].

[11] S. Hemming and L. Thorlacius, Thermodynamics of Large AdS Black Holes, JHEP 11 (2007) 086 [arXiv:0709.3738] [INSPIRE].

[12] R. Gregory, S.F. Ross and R. Zegers, Classical and quantum gravity of brane black holes, JHEP 09 (2008) 029 [arXiv:0802.2037] [InSPIRE].

[13] J.V. Rocha, Evaporation of large black holes in AdS: Coupling to the evaporon, JHEP 08 (2008) 075 [arXiv: 0804.0055] [INSPIRE]. 
[14] Z.-H. Li, B. Hu and R.-G. Cai, A Note on Self-gravitating Radiation in AdS Spacetime, Phys. Rev. D 77 (2008) 104032 [arXiv:0804.3233] [INSPIRE].

[15] K. Saraswat and N. Afshordi, Quantum Nature of Black Holes: Fast Scrambling versus Echoes, JHEP 04 (2020) 136 [arXiv: 1906.02653] [INSPIRE].

[16] R.B. Mann and S.N. Solodukhin, Universality of quantum entropy for extreme black holes, Nucl. Phys. B 523 (1998) 293 [hep-th/9709064] [INSPIRE].

[17] A. Sen, Logarithmic Corrections to Rotating Extremal Black Hole Entropy in Four and Five Dimensions, Gen. Rel. Grav. 44 (2012) 1947 [arXiv:1109.3706] [INSPIRE].

[18] A. Ashtekar, Lectures on non-perturbative canonical gravity, World Scientific, Singapore (1991).

[19] T.R. Govindarajan, R.K. Kaul and V. Suneeta, Logarithmic correction to the Bekenstein-Hawking entropy of the BTZ black hole, Class. Quant. Grav. 18 (2001) 2877 [gr-qc/0104010] [INSPIRE].

[20] D. Birmingham and S. Sen, An exact black hole entropy bound, Phys. Rev. D 63 (2001) 047501 [hep-th/0008051] [INSPIRE].

[21] T. Jacobson, Thermodynamics of space-time: The Einstein equation of state, Phys. Rev. Lett. 75 (1995) 1260 [gr-qc/9504004] [INSPIRE].

[22] M. Faizal, A. Ashour, M. Alcheikh, L. Alasfar, S. Alsaleh and A. Mahroussah, Quantum fluctuations from thermal fluctuations in Jacobson formalism, Eur. Phys. J. C 77 (2017) 608 [arXiv: 1710.06918] [INSPIRE].

[23] S. Das, P. Majumdar and R.K. Bhaduri, General logarithmic corrections to black hole entropy, Class. Quant. Grav. 19 (2002) 2355 [hep-th/0111001] [INSPIRE].

[24] S. Upadhyay, B. Pourhassan and H. Farahani, P-V criticality of first-order entropy corrected AdS black holes in massive gravity, Phys. Rev. D 95 (2017) 106014 [arXiv:1704.01016] [INSPIRE].

[25] A. Jawad, Consequences of Thermal Fluctuations of Well-Known Black Holes in Modified Gravity, Class. Quant. Grav. 37 (2020) 185020 [arXiv: 2008.11033] [inSPIRE].

[26] B. Pourhassan, PV criticality of the second order quantum corrected Horava-Lifshitz black hole, Eur. Phys. J. C 79 (2019) 740 [arXiv:1905.13032] [INSPIRE].

[27] J. Sadeghi, B. Pourhassan and M. Rostami, P-V criticality of logarithm-corrected dyonic charged AdS black holes, Phys. Rev. D 94 (2016) 064006 [arXiv: 1605.03458] [INSPIRE].

[28] B. Pourhassan, S. Dey, S. Chougule and M. Faizal, Quantum corrections to a finite temperature BIon, Class. Quant. Grav. 37 (2020) 135004 [arXiv:1905.03624] [INSPIRE].

[29] B. Pourhassan, S. Upadhyay, H. Saadat and H. Farahani, Quantum gravity effects on Hořava-Lifshitz black hole, Nucl. Phys. B 928 (2018) 415 [arXiv:1705.03005] [InSPIRE].

[30] B. Pourhassan, M. Faizal, Z. Zaz and A. Bhat, Quantum fluctuations of a BTZ black hole in massive gravity, Phys. Lett. B 773 (2017) 325 [arXiv:1709.09573] [INSPIRE].

[31] B. Pourhassan, A. Övgün and İ. Sakall, $P V$ criticality of Achúcarro-Ortiz black hole in the presence of higher order quantum and GUP corrections, Int. J. Geom. Meth. Mod. Phys. 17 (2020) 2050156 [arXiv: 1811.02193] [INSPIRE].

[32] A. Chatterjee and A. Ghosh, Exponential Corrections to Black Hole Entropy, Phys. Rev. Lett. 125 (2020) 041302 [arXiv:2007.15401] [INSPIRE]. 
[33] A. Dabholkar, J. Gomes and S. Murthy, Nonperturbative black hole entropy and Kloosterman sums, JHEP 03 (2015) 074 [arXiv: 1404.0033] [INSPIRE].

[34] A. Dabholkar, J. Gomes and S. Murthy, Localization $\&$ Exact Holography, JHEP 04 (2013) 062 [arXiv:1111.1161] [INSPIRE].

[35] S. Murthy and B. Pioline, A Farey tale for $N=4$ dyons, JHEP 09 (2009) 022 [arXiv: 0904.4253] [INSPIRE].

[36] R.C. Myers and M.J. Perry, Black Holes in Higher Dimensional Space-Times, Annals Phys. 172 (1986) 304 [INSPIRE].

[37] D. Astefanesei, M.J. Rodriguez and S. Theisen, Thermodynamic instability of doubly spinning black objects, JHEP 08 (2010) 046 [arXiv:1003.2421] [INSPIRE].

[38] M. Garbiso and M. Kaminski, Hydrodynamics of simply spinning black holes 83 hydrodynamics for spinning quantum fluids, JHEP 12 (2020) 112 [arXiv:2007.04345] [INSPIRE].

[39] H. Saadat and A. Pourdarvish, Myerse-Perry Black Holes with Logarithmic Correction, Int. J. Theor. Phys. 53 (2014) 3014 [InSPIRE].

[40] M. Stein, Perturbative Construction of Stationary Randall-Sundrum II Black Holes on a 5-Brane, JHEP 09 (2016) 067 [arXiv: 1605.06128] [INSPIRE].

[41] B.P. Dolan, Intrinsic curvature of thermodynamic potentials for black holes with critical points, Phys. Rev. D 92 (2015) 044013 [arXiv: 1504.02951] [InSPIRE].

[42] D.F. Litim and K. Nikolakopoulos, Quantum gravity effects in Myers-Perry space-times, JHEP 04 (2014) 021 [arXiv: 1308.5630] [INSPIRE].

[43] B. de Lima Bernardo, Unraveling the role of coherence in the first law of quantum thermodynamics, Phys. Rev. E 102 (2020) 062152.

[44] A. Teixidó-Bonfill, A. Ortega and E. Martín-Martínez, First law of quantum field thermodynamics, Phys. Rev. A 102 (2020) 052219 [arXiv: 2008.09146] [InSPIRE].

[45] A. Ortega, E. McKay, A.M. Alhambra and E. Martín-Martínez, Work distributions on quantum fields, Phys. Rev. Lett. 122 (2019) 240604 [arXiv:1902.03258] [INSPIRE].

[46] S.L. Braunstein and A.K. Pati, Quantum information cannot be completely hidden in correlations: Implications for the black-hole information paradox, Phys. Rev. Lett. 98 (2007) 080502 [gr-qc/0603046] [INSPIRE].

[47] D.N. Page, Average entropy of a subsystem, Phys. Rev. Lett. 71 (1993) 1291 [gr-qc/9305007] [INSPIRE].

[48] J. Liu, H. Yuan, X.-M. Lu and X. Wang, Quantum Fisher information matrix and multiparameter estimation, J. Phys. A 53 (2020) 023001 [arXiv:1907.08037] [InSPIRE].

[49] J. Goold, M. Huber, A. Riera, L. del Rio and P. Skrzypczyk, The role of quantum information in thermodynamics - a topical review, J. Phys. A 49 (2016) 143001.

[50] S. Lloyd and J. Preskill, Unitarity of black hole evaporation in final-state projection models, JHEP 08 (2014) 126 [arXiv:1308.4209] [INSPIRE].

[51] D. Marolf and J. Polchinski, Gauge/Gravity Duality and the Black Hole Interior, Phys. Rev. Lett. 111 (2013) 171301 [arXiv: 1307.4706] [INSPIRE].

[52] S. Carlip, Logarithmic corrections to black hole entropy from the Cardy formula, Class. Quant. Grav. 17 (2000) 4175 [gr-qc/0005017] [INSPIRE]. 
[53] J.-l. Jing and M.-L. Yan, Statistical entropy of the static dilaton black holes from the Cardy formulas, Phys. Rev. D 63 (2001) 024003 [gr-qc/0005105] [inSPIRE].

[54] M. Hassaine, Cardy-like formula for the Schwarzschild black hole entropy, Phys. Rev. D 101 (2020) 084028 [arXiv: 1909.06896] [INSPIRE].

[55] M. Honda, Quantum Black Hole Entropy from 4 d Supersymmetric Cardy formula, Phys. Rev. D 100 (2019) 026008 [arXiv: 1901.08091] [INSPIRE].

[56] B. Chen and J. Long, Real-time Correlators and Hidden Conformal Symmetry in Kerr/CFT Correspondence, JHEP 06 (2010) 018 [arXiv: 1004.5039] [INSPIRE].

[57] S.M. Noorbakhsh and M. Ghominejad, Ultra-Spinning Gauged Supergravity Black Holes and their Kerr/CFT Correspondence, Phys. Rev. D 95 (2017) 046002 [arXiv:1611.02324] [INSPIRE].

[58] A. Pourdarvish and B. Pourhassan, Statistics of Myerse-Perry Black Holes, Int. J. Theor. Phys. 53 (2014) 136 [INSPIRE].

[59] B. Chen, S.-x. Liu and J.-j. Zhang, Thermodynamics of Black Hole Horizons and Kerr/CFT Correspondence, JHEP 11 (2012) 017 [arXiv:1206.2015] [INSPIRE].

[60] Z. Fei, N. Freitas, V. Cavina, H.T. Quan and M. Esposito, Work statistics across a quantum phase transition, Phys. Rev. Lett. 124 (2020) 170603 [arXiv:2002.07860] [INSPIRE].

[61] B.-B. Wei, Quantum work relations and response theory in parity-time-symmetric quantum systems, Phys. Rev. E 97 (2018) 012114 [arXiv:1711.00586] [InSPIRE].

[62] J. Salmilehto, P. Solinas and M. Möttönen, Quantum Driving and Work, Phys. Rev. E 89 (2014) 052128 [arXiv: 1401.4440] [INSPIRE].

[63] P. Talkner, E. Lutz and P. Hänggi, Fluctuation theorems: Work is not an observable, Phys. Rev. E 75 (2007) 050102.

[64] G.E. Crooks, Nonequilibrium Measurements of Free Energy Differences for Microscopically Reversible Markovian Systems, J. Statist. Phys. 90 (1998) 1481.

[65] G.E. Crooks, Entropy production fluctuation theorem and the nonequilibrium work relation for free energy differences, Phys. Rev. E 60 (1999) 2721.

[66] C. Jarzynski, Nonequilibrium Equality for Free Energy Differences, Phys. Rev. Lett. 78 (1997) 2690 [cond-mat/9610209] [INSPIRE].

[67] C. Jarzynski, Microscopic Analysis of Clausius-Duhem Processes, J. Statist. Phys. 96 (1999) 415.

[68] S. Iso, S. Okazawa and S. Zhang, Non-equilibrium fluctuations of black hole horizons and the generalized second law, Phys. Lett. B 705 (2011) 152 [arXiv:1008.1184] [INSPIRE].

[69] S. Iso and S. Okazawa, Stochastic Equations in Black Hole Backgrounds and Non-equilibrium Fluctuation Theorems, Nucl. Phys. B 851 (2011) 380 [arXiv:1104.2461] [INSPIRE].

[70] H. Dimov, R.C. Rashkov and T. Vetsov, Thermodynamic information geometry and complexity growth of a warped AdS black hole and the warped $A d S_{3} / C F T_{2}$ correspondence, Phys. Rev. D 99 (2019) 126007 [arXiv:1902.02433] [INSPIRE].

[71] T. Vetsov, Information Geometry on the Space of Equilibrium States of Black Holes in Higher Derivative Theories, Eur. Phys. J. C 79 (2019) 71 [arXiv:1806.05011] [INSPIRE]. 
[72] A. Sheykhi, F. Naeimipour and S.M. Zebarjad, Phase transition and thermodynamic geometry of topological dilaton black holes in gravitating logarithmic nonlinear electrodynamics, Phys. Rev. D 91 (2015) 124057 [InSPIRE].

[73] G.-Q. Li and J.-X. Mo, Phase transition and thermodynamic geometry of $f(R)$ AdS black holes in the grand canonical ensemble, Phys. Rev. D 93 (2016) 124021 [arXiv:1605.09121] [INSPIRE].

[74] S.-W. Wei and Y.-X. Liu, Insight into the Microscopic Structure of an AdS Black Hole from a Thermodynamical Phase Transition, Phys. Rev. Lett. 115 (2015) 111302 [Erratum ibid. 116 (2016) 169903] [arXiv: 1502.00386] [INSPIRE].

[75] S.-W. Wei, Y.-X. Liu and R.B. Mann, Repulsive Interactions and Universal Properties of Charged Anti-de Sitter Black Hole Microstructures, Phys. Rev. Lett. 123 (2019) 071103 [arXiv: 1906.10840] [INSPIRE].

[76] M. Dehghani and M. Badpa, Phase transition and geometrical thermodynamics of energy-dependent dilatonic BTZ black holes with power-law electrodynamics, PTEP 2020 (2020) 033E03.

[77] M. Dehghani, Nonlinearly charged AdS black hole solutions in three-dimensional massive gravity's rainbow, Phys. Lett. B 803 (2020) 135335 [INSPIRE].

[78] S. Soroushfar and S. Upadhyay, Phase transition of a charged AdS black hole with a global monopole through geometrical thermodynamics, Phys. Lett. B 804 (2020) 135360 [arXiv: 2003.06714] [INSPIRE].

[79] A. Ghosh and C. Bhamidipati, Thermodynamic geometry for charged Gauss-Bonnet black holes in AdS spacetimes, Phys. Rev. D 101 (2020) 046005 [arXiv:1911.06280] [INSPIRE].

[80] J. Suresh, R. Tharanath, N. Varghese and V.C. Kuriakose, The thermodynamics and thermodynamic geometry of the Park black hole, Eur. Phys. J. C 74 (2014) 2819 [arXiv: 1403.4710] [INSPIRE].

[81] S. Soroushfar, R. Saffari and S. Upadhyay, Thermodynamic geometry of a black hole surrounded by perfect fluid in Rastall theory, Gen. Rel. Grav. 51 (2019) 130 [arXiv: 1908.02133] [INSPIRE].

[82] G. Ruppeiner, Riemannian geometry in thermodynamic fluctuation theory, Rev. Mod. Phys. 67 (1995) 605 [Erratum ibid. 68 (1996) 313] [INSPIRE].

[83] G. Ruppeiner, Thermodynamics: A riemannian geometric model, Phys. Rev. A 20 (1979) 1608.

[84] F. Weinhold, Metric geometry of equilibrium thermodynamics. II. Scaling, homogeneity, and generalized Gibbs-Duhem relations, J. Chem. Phys. 63 (1975) 2484.

[85] F. Weinhold, Metric geometry of equilibrium thermodynamics, J. Chem. Phys. 63 (1975) 2479.

[86] H. Quevedo and A. Sanchez, Geometrothermodynamics of asymptotically de Sitter black holes, JHEP 09 (2008) 034 [arXiv:0805.3003] [INSPIRE].

[87] H. Quevedo, Geometrothermodynamics, J. Math. Phys. 48 (2007) 013506 [physics/0604164] [INSPIRE].

[88] S.H. Hendi, S. Panahiyan, B. Eslam Panah and M. Momennia, A new approach toward geometrical concept of black hole thermodynamics, Eur. Phys. J. C 75 (2015) 507 [arXiv: 1506.08092] [INSPIRE]. 
[89] S.H. Hendi, B. Eslam Panah and S. Panahiyan, Massive charged BTZ black holes in asymptotically (a)dS spacetimes, JHEP 05 (2016) 029 [arXiv: 1604.00370] [INSPIRE].

[90] S.H. Hendi, A. Sheykhi, S. Panahiyan and B. Eslam Panah, Phase transition and thermodynamic geometry of Einstein-Maxwell-dilaton black holes, Phys. Rev. D 92 (2015) 064028 [arXiv: 1509.08593] [INSPIRE].

[91] S.H. Hendi, S. Panahiyan, B.E. Panah and Z. Armanfard, Phase transition of charged Black Holes in Brans-Dicke theory through geometrical thermodynamics, Eur. Phys. J. C 76 (2016) 396 [arXiv: 1511.00598] [INSPIRE].

[92] S.A. Hosseini Mansoori and B. Mirza, Geometrothermodynamics as a singular conformal thermodynamic geometry, Phys. Lett. B 799 (2019) 135040 [arXiv:1905. 01733] [INSPIRE].

[93] S.A. Hosseini Mansoori, M. Rafiee and S.-W. Wei, Universal criticality of thermodynamic curvatures for charged AdS black holes, Phys. Rev. D 102 (2020) 124066 [arXiv: 2007.03255] [INSPIRE]. 